\title{
AVALIAÇÃO DO GOSTO AMARGO DA BEBIDA DE CAFÉ (Coffea arabica L.) ORGÂNICO POR MEIO DA ANÁLISE TEMPO-INTENSIDADE ${ }^{1}$
}

\author{
Aline Fonseca DA SILVA², Valéria Paula Rodrigues MINIM², José Benício Paes CHAVES², \\ Paulo César STRINGHETA², Milene Moreira RIBEIRO²
}

\section{RESUMO}

Os consumidores estão preocupados em adquirir produtos que atendam as questões ambientais, sociais e conseqüentemente sejam saudáveis, contribuindo para o crescimento da comercialização de produtos orgânicos em todo mundo. O café, produto tradicionalmente cultivado no Brasil e de grande aceitação ( $70 \%$ da população brasileira consome café diariamente), é um dos produtos cultivados sob manejo orgânico. Este trabalho teve por objetivo avaliar as características temporais do gosto amargo na bebida de café orgânico. Foram avaliadas quatro marcas de café orgânico (ORG-1, ORG-2, ORG-3 e ORG-4) e uma marca de café convencional (CON) por meio da Análise Tempo-Intensidade. Sete provadores selecionados e treinados avaliaram as amostras de café utilizando o programa "Sistema de Coleta de Dados Tempo-Intensidade-SCDTI" para Windows. Os resultados obtidos foram estatisticamente analisados por Análise de Variância (ANOVA), teste de Duncan e Análise de Componente Principal (ACP). A marca ORG-3 apresentou maior intensidade máxima (Imax), apresentando diferença estatística significativa $(\mathrm{p} \leq 0,05)$ em relação as demais amostras para a percepção desse atributo. Entre as amostras ORG-1, ORG-2, ORG-4 e CON não houve diferença estatística significativa $(\mathrm{p} \geq 0,05)$ em relação aos seis parâmetros avaliados.

Palavras-chave: café; orgânico; Análise Tempo-Intensidade; análise sensorial.

\section{SUMMARY}

EVALUATION OF THE BITTER TASTE IN ORGANIC COFFEE (Coffea arabica L.) BEVERAGE USING TIME-INTENSITY ANALYSIS. Nowadays consumers are concerned with purchasing products which are environmentally - friendly and socially - oriented and, consequently, healthy. Thus, the commercialization of organic products has expanded worldwide. Coffee is traditionally cultivated in Brazil and widely accepted $(70 \%$ of the population drink coffee daily). It is also organically cultivated. This work aimed to evaluate the temporal characteristics of the bitter taste in organic coffee drink. Four organic coffee brands (ORG-1, ORG-2, ORG-3 and ORG-4) and one conventional (CON) brand were evaluated by Time-Intensity Analysis. Seven selected and trained tasters evaluated the coffee samples using the program "Sistema de Coleta de Dados Tempo-Intensidade-SCDTI" (TIME-INTENSITY DATA COLLECTION SYSTEM-TIDCS) for Windows. The results were statistically analyzed by Analysis of Variance (ANOVA), Duncan's tests and Principal Component Analysis (PCA). The ORG-3 brand showed higher maximum intensity (Imax), presenting a significant difference ( $\mathrm{p} \leq 0.05$ ), compared to the other samples. No significant differences were found for ORG-1, ORG-2, ORG-4 and CON ( $\geq 20.05)$ regarding the six appraised parameters. Keywords: coffee; organic; time-intensity analysis; sensory analysis.

\section{1 - INTRODUÇÃO}

Da produção ao consumo, o café passa por um longo ciclo que pode ser afetado por inúmeros fatores alterando aparência, aroma e sabor da bebida. Provadores de café em todo o mundo apontam a melhoria da qualidade do grão, proveniente de sistemas de produção orgânica, como aromas e sabores específicos da bebida, observando-se todos os cuidados nas fases de pré e pós-colheita que o cultivo orgânico exige [13].

O sabor característico do café como bebida é proveniente do grão, estando diretamente relacionado com as variedades e influenciado por tratos agrícolas, processos de secagem, fermentação, torrefação, moagem e envase [2].

A Análise Tempo-Intensidade tem sido usada como ferramenta fundamental para pesquisa com doçura [1, $4,11]$ e amargor $[9,10]$.

\footnotetext{
1. Recebido para publicação em 01/10/2003. Aceito para publicação em 06/07/2004 (001226).

2. Universidade Federal de Viçosa, Departamento de Tecnologia de Alimentos. - Viçosa - MG. CEP: 36571-O0O. Tel: (31) 3899-2226. E-mail: alinefonseca3@yahoo.com.br

* A quem a correspondência deve ser enviada.
}

Diferentes estímulos sensoriais possuem uma característica única no curso da percepção, que é o aumento da percepção seguido por uma intensidade máxima e culminando na sua extinção [7].

O método Tempo-Intensidade é muito importante para a quantificação de diferenças temporais em características sensoriais, tais como gostos básicos, e percepções de sabores como adstringência [8]. E oferece melhor aproximação do "mundo real" do fenômeno associado à percepção de um estímulo que ocorre na cavidade oral com o passar do tempo, que é impossível nos procedimentos de tempo livre [3].

MONTEIRO [10] utilizou a metodologia Tempo-Intensidade visando avaliar o gosto amargo, sabor queimado e sabor fermentado em amostras de café provenientes de três classes e submetidas a diferentes torras, sendo este o único registro sobre o uso da técnica Tempo-Intensidade com a bebida de café.

A percepção do aroma, do sabor e da textura é um fenômeno dinâmico e não estático, sendo de suma importância à aplicação da análise tempo-intensidade como forma de avaliação de um alimento [10], que através da associação da percepção humana com recursos da informática permite obter informações sobre qualquer característica sensorial pré-estabelecida das amostras avaliadas, como por exemplo, velocidade, tempo de percepção e intensidade de um estímulo [5]. 
O gosto amargo é uma das características sensoriais mais marcante em café podendo afetar na aceitação deste produto.

Com base no que foi exposto anteriormente, o presente trabalho teve por objetivo avaliar o gosto amargo por meio da técnica Tempo-Intensidade em amostras de café orgânico e convencional.

\section{2 - MATERIAL E MÉTODOS}

\section{1 - Amostras}

Foram avaliadas quatro marcas comerciais de café orgânico torrado e moído (ORG-1, ORG-2, ORG-3, ORG4) e uma de café convencional torrado e moído (CON), sendo que os cafés ORG-4 e CON foram da mesma marca. As amostras de cada marca foram provenientes do mesmo tipo de torra e lote de fabricação. As marcas de café utilizadas nesse trabalho são encontradas principalmente na região Sudeste, em supermercados de grande porte e lojas especializadas em produtos orgânicos. Não foi possivel estabelecer a classificação da bebida, pois, nas embalagens constava somente a data de validade, lote de fabricação e o selo de certificação.

\section{2 - Condições do teste, preparo e apresentação das amostras}

De acordo com a recomendação de CARVALHO, CHAGAS \& CHALFOUN [6], as amostras foram preparadas com água deionizada e pó de café na proporção de $6 \%$, ou seja, para cada $30 \mathrm{~g}$ de pó de café foi utilizado $500 \mathrm{~mL}$ de água deionizada. A bebida foi preparada em uma cafeteira elétrica e armazenada em garrafa térmica por uma hora no máximo para que a bebida não fosse servida em temperatura inadequada. As amostras foram servidas em xícaras de fundo preto, codificados com três dígitos, contendo aproximadamente $30 \mathrm{~mL}$. O café foi servido sem adição de sacarose ou qualquer tipo de adoçante.

\section{3 - Sistema de Coleta de Dados Tempo-Intensida- de - SCDTI}

O programa utilizado para a Análise Tempo-Intensidade foi desenvolvido no Laboratório de Análise Sensorial da Faculdade de Engenharia de AlimentosUNICAMP denominado Sistema de Coleta de Dados Tempo-Intensidade-SCDTI versão 1.0.

O SCDTI opera em ambiente Windows, e consiste em um programa para coleta e manipulação de dados Tempo-Intensidade, no qual o usuário indica utilizando "mouse" a atual intensidade do estímulo a ser coletado. No decorrer da análise, são apresentadas mensagens de aviso do início de novas etapas, com instruções ao provador sobre a ação a ser realizada.

O tempo estipulado para a realização da análise de Tempo-Intensidade foi de vinte segundos, determinado por uma média de tempo dos provadores. A marca de café orgânico ORG-2 foi utilizada para esse fim, onde os provadores o avaliaram em três repetições o tempo do início do estímulo amargo na boca e o desapareci- mento total do mesmo foram marcados com um cronômetro, posteriormente retirou-se a média do tempo.

\section{4 - Recrutamento e pré-seleção de provadores}

Foram recrutados os provadores que já tinham participado da Análise Descritiva Quantitativa destas amostras de café, pelo fato de já serem treinados, sendo selecionados aqueles que possuíam interesse em realizar os testes, disponibilidade de tempo para as análises e pela habilidade com o computador.

\section{5 - Determinação do atributo e treinamento dos provadores}

A equipe sensorial a partir de um consenso determinou que o gosto amargo seria o atributo de maior relevância para representação das amostras de café. Após a escolha do atributo a ser avaliado, os provadores passaram por algumas sessões de treinamento para padronização do método que consistiu na familiarização com o programa SCDTI, para que os provadores pudessem além de memorizar a sensação percebida, aprimorassem a coordenação motora e utilizassem o mouse em sincronia com a percepção do atributo.

Foi utilizado o padrão de referência para gosto amargo fraco a bebida café Bom Dia Expresso e gosto amargo forte a bebida café orgânico Native.

\section{6 - Seleção final dos provadores}

A seleção da equipe final de provadores foi realizada por meio de um teste preliminar com as amostras de café ORG-4, ORG-2 e CON. As amostras foram avaliadas em três repetições, sendo apresentadas de forma monádica.

Os resultados obtidos por provador e para cada parâmetro da curva Tempo-Intensidade foram analisados pela Análise de Variância Univariada (ANOVA), com as fontes de variação amostra e repetição. Foram dispensados os provadores que obtiveram probabilidade para $\mathrm{F}_{\text {amostra }}$ maior ou igual a 0,50 ou probabilidade para $\mathrm{F}_{\text {repeticăo }}$ menor ou igual a 0,05 em pelo menos um dos parâmetros [10].

\section{7 - Avaliação das amostras e parâmetros da cur- va Tempo-Intensidade}

As amostras foram apresentadas de forma monádica e aleatorizadas por sorteio, com três repetições. Foram servidas em xícaras de fundo preto, com aproximadamente $30 \mathrm{~mL}$ de café.Todas as instruções foram dadas pelo programa SCDTI, para que o provador colocasse a amostra na boca, engolisse a amostra dentro de um determinado tempo previamente estipulado. A escala utilizada para a análise foi de nove pontos, sendo 0 referente a nenhum e 9 referente a forte.

Os parâmetros da curva avaliados foram TImax (tempo de intensidade máxima), Imax (intensidade máxima), Platô (tempo de duração da intensidade máxima), Área (área sob a curva), Td (tempo correspondente ao ponto onde a intensidade máxima começa a declinar) e Ttot (tempo total de duração do estímulo). 


\section{8 - Análise dos resultados}

Foi realizada uma Análise de Variância Univariada (ANOVA) para os dados obtidos dos parâmetros citados acima, utilizando-se amostra e provador como fontes de variação e interação para cada parâmetro. O teste de comparação de médias (Duncan) para as amostras de café foi realizado. As análises estatísticas foram realizadas utilizando-se o programa estatístico SAS (Statistical Analysis System) [12], versão 8.0, licenciado para Universidade Federal de Viçosa.

\section{3 - RESULTADOS E DISCUSSÃO}

\section{1 - Recrutamento e pré-seleção de provadores}

Dos dez provadores selecionados e treinados que participaram da Análise Descritiva Quantitativa, oito provadores (quatro homens e quatro mulheres) foram pré-selecionados devido ao interesse, disponibilidade e habilidade com o computador.

\section{2 - Seleção final dos provadores}

Os valores de probabilidade de $\mathrm{F}_{\text {amostra }}$, que indica o poder do provador em discriminar as amostras, e a probabilidade de $\mathrm{F}_{\text {repeticão, }}$ que determina a repetibilidade dos provadores entre as amostras para cada parâmetro da curva, estão apresentados nas Tabelas 1 e 2, respectivamente.

TABELA 1. Níveis de probabilidade de $\mathrm{F}_{\text {amostra }}$ dos provadores para os parâmetros da curva Tempo-Intensidade.

\begin{tabular}{ccccccc}
\hline Provador & TI max & Imax & Área & Td & Platô & Ttot \\
\hline 1 & 0,48 & 0,27 & 0,15 & 0,10 & 0,10 & 0,43 \\
2 & 0,34 & 0,68 & 0,42 & 0,11 & 0,17 & 0,44 \\
3 & 0,03 & 0,44 & 0,46 & 0,23 & 0,36 & 0,26 \\
4 & 0,25 & 0,10 & 0,12 & 0,31 & 0,44 & 0,48 \\
5 & 0,13 & 0,05 & 0,02 & 0,06 & 0,49 & 0,45 \\
6 & 0,08 & 0,06 & 0,05 & 0,30 & 0,23 & 0,46 \\
7 & 0,33 & 0,23 & 0,41 & 0,27 & 0,23 & 0,21 \\
8 & 0,11 & 0,00 & 0,05 & 0,40 & 0,18 & 0,49 \\
\hline
\end{tabular}

Probabilidade maior ou igual a 0,50 indica que o provador não está contribuindo para a discriminacão entre as amostras. TImax: tempo de intensidade maxima, Imax: intensidade maxima, Platô: tempo de duração da intensidade maxima, Area: área ob a curva, Td: tempo correspondente ao ponto onde a intensidade máxima começa a declinar; Ttot: tempo total de duração do estímulo

TABELA 2. Níveis de probabilidade de $F_{\text {repeticăo }}$ dos provadores para os parâmetros da curva Tempo-Intensidade.

\begin{tabular}{ccccccc}
\hline Provador & TI max & Imax & Área & Td & Platô & Ttot \\
\hline 1 & 0,96 & 0,11 & 0,12 & 0,90 & 0,51 & 0,44 \\
2 & 0,60 & 0,37 & 0,34 & 0,31 & 0,36 & 0,11 \\
3 & 0,14 & 0,51 & 0,51 & 0,57 & 0,11 & 0,48 \\
4 & 0,45 & 0,52 & 0,32 & 0,82 & 0,46 & 0,46 \\
5 & 0,37 & 0,28 & 0,84 & 0,34 & 0,46 & 0,31 \\
6 & 0,68 & 0,50 & 0,80 & 0,18 & 0,25 & 0,46 \\
7 & 0,38 & 0,47 & 0,37 & 0,47 & 0,88 & 0,33 \\
8 & 0,94 & 0,08 & 0,56 & 0,41 & 0,90 & 0,77 \\
\hline
\end{tabular}

Probabilidade menor ou igual a 0,05 indica que a repetibilidade do provador não estã satisfatória para o critério de corte estabelecido. TImax: tempo de intensidade máxima, Imax: intensidade máxima, Area: área sob a curva, Td: tempo correspondente ao ponto onde a intensidade máxima começa a declinar; Platô: tempo de duração da intensidade máxima, Ttot: tempo total de duração do estímulo.
Como o provador 2 apresentou probabilidade de $\mathrm{F}_{\text {amostra }}$ igual a 0,68 foi dispensado e os outros sete provadores (três mulheres e quatro homens) foram selecionados para participarem da avaliação final das amostras da bebida de café para o atributo gosto amargo.

\section{3 - Avaliação das amostras}

A representação gráfica dos resultados obtidos da Análise de Componentes Principais em relação ao gosto amargo das amostras de café está representada na Figura 1.

O primeiro componente principal explica 99,7\% da variação total entre as amostras e está associado as variáveis Imax (Intensidade Máxima) e Área (Tabela 3), portanto estes são os parâmetros mais importantes para o gosto amargo, já o segundo componente principal explica $0,2 \%$ da variação e está associado ao tempo correspondente ao ponto, onde a intensidade máxima do gosto amargo começa a declinar (Td). Os dois componentes principais explicam $99,9 \%$ da variância entre as amostras de café.

O primeiro componente principal sugere que a marca de café orgânico ORG-3 apresenta maior intensidade máxima e a área sob a curva e para o gosto amargo, ao contrário das demais amostras de café que apresentam esses parâmetros em menor intensidade.
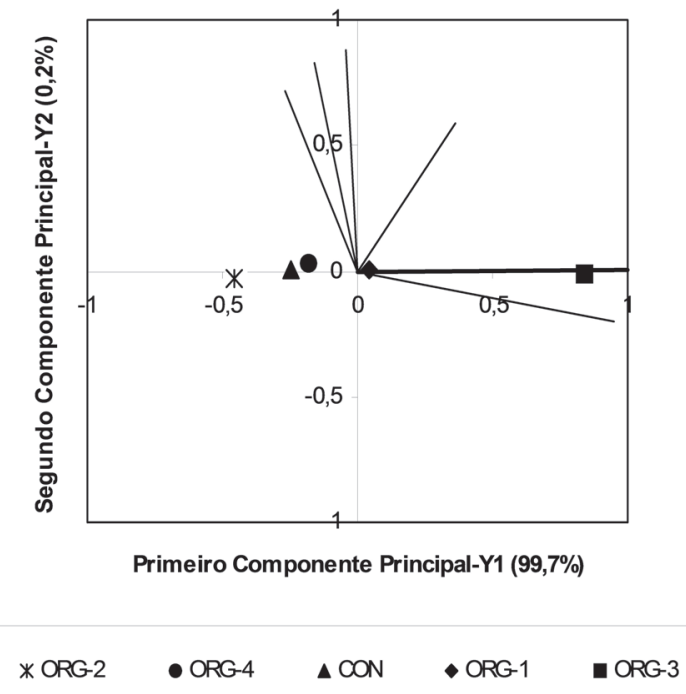

FIGURA 1. Análise de Componentes Principais dos parâmetros da curva Tempo-Intensidade para as amostras de café orgânico e convencional.

A análise de variância dos resultados mostrou que no parâmetro Área e Td (tempo correspondente ao ponto onde a intensidade máxima começa a declinar) houve interação significativa $(\mathrm{p} \leq 0,05)$ entre amostra e provador e para os demais parâmetros não houve interação significativa $(\mathrm{p} \geq 0,05)$. Sendo assim, para Área e $\mathrm{Td}$ foi realizado o teste $\mathrm{F}$ para efeito de tratamentos, utilizando-se o quadrado médio da interação como denominador. 
TABELA 3. Coeficientes de Correlação de Pearson entre os parâmetros da curva Tempo-Intensidade e os dois componentes principais.

\begin{tabular}{|c|c|c|c|c|}
\hline \multirow{3}{*}{ PARÂMETROS } & \multicolumn{4}{|c|}{ Componentes Principais } \\
\hline & \multicolumn{2}{|c|}{ Y1 } & \multicolumn{2}{|c|}{ Y2 } \\
\hline & $r$ & $\mathrm{p}$ & $r$ & $\mathrm{p}$ \\
\hline TImax & $-0,27$ & 0,66 & 0,71 & 0,18 \\
\hline Imax & 0,95 & 0,01 & $-0,20$ & 0,74 \\
\hline Área & 1,00 & 0,00 & 0,001 & 0,99 \\
\hline $\mathrm{Td}$ & $-0,04$ & 0,94 & 0,88 & 0,05 \\
\hline Platô & 0,36 & 0,55 & 0,59 & 0,30 \\
\hline Ttot & $-0,16$ & 0,80 & 0,83 & 0,08 \\
\hline
\end{tabular}

r: Coeficiente de Correlação; p: nível de significância; TImax: tempo de intensidade máxima, Imax: intensidade máxima, Área: área sob a curva, Td: tempo correspondente ao ponto onde a intensidade máxima começa a declinar; Platô: tempo de duração da intensidade máxima, Ttot: tempo total de duração do estímulo.

Para todos os parâmetros da curva de Tempo-Intensidade o efeito provador foi significativo e em relação às amostras de café houve efeito significativo $(\mathrm{p} \leq 0,05)$ para os parâmetros de intensidade máxima (Imax) e Área.

Na Tabela 4 encontra-se o resultado do teste de comparação de médias (Duncan) e pode-se observar que o café ORG-3 apresentou maior intensidade máxima e área confirmando assim as informações sugeridas pela Análise de Componentes Principais.

TABELA 4. Médias dos parâmetros da curva Tempo-Intensidade para as amostras de café.

\begin{tabular}{cccccc}
\hline \multirow{2}{*}{ PARÂMETROS } & \multicolumn{5}{c}{ AMOSTRAS } \\
\cline { 2 - 6 } & ORG-1 & ORG-2 & ORG-3 & ORG-4 & CON \\
\hline TImax & $14,34 \mathrm{a}$ & $13,94 \mathrm{a}$ & $13,84 \mathrm{a}$ & $14,38 \mathrm{a}$ & $13,91 \mathrm{a}$ \\
Imax & $5,65 \mathrm{~b}$ & $5,49 \mathrm{~b}$ & $7,99 \mathrm{a}$ & $5,62 \mathrm{~b}$ & $5,61 \mathrm{~b}$ \\
Área & $65,73 \mathrm{~b}$ & $50,71 \mathrm{~b}$ & $89,50 \mathrm{a}$ & $59,12 \mathrm{~b}$ & $57,09 \mathrm{~b}$ \\
Td & $17,58 \mathrm{a}$ & $16,59 \mathrm{a}$ & $16,88 \mathrm{a}$ & $17,40 \mathrm{a}$ & $17,32 \mathrm{a}$ \\
Platô & $3,24 \mathrm{a}$ & $2,65 \mathrm{a}$ & $3,14 \mathrm{a}$ & $3,02 \mathrm{a}$ & $3,36 \mathrm{a}$ \\
Ttot & $28,11 \mathrm{a}$ & $27,96 \mathrm{a}$ & $28,24 \mathrm{a}$ & $29,42 \mathrm{a}$ & $28,64 \mathrm{a}$ \\
\hline
\end{tabular}

Médias seguidas pela mesma letra na linha não diferem significativamente entre $s$ pelo teste de Duncan ao nivel de 5\% de probabilidade. TImax: tempo de intensidade máxima, Imax: intensidade máxima, Área: área sob a curva, Td: tempo correspondente ao ponto onde a intensidade máxima começa a declinar; Platô: tempo de duração da intensidade máxima, Ttot: tempo total de duração do estímulo.

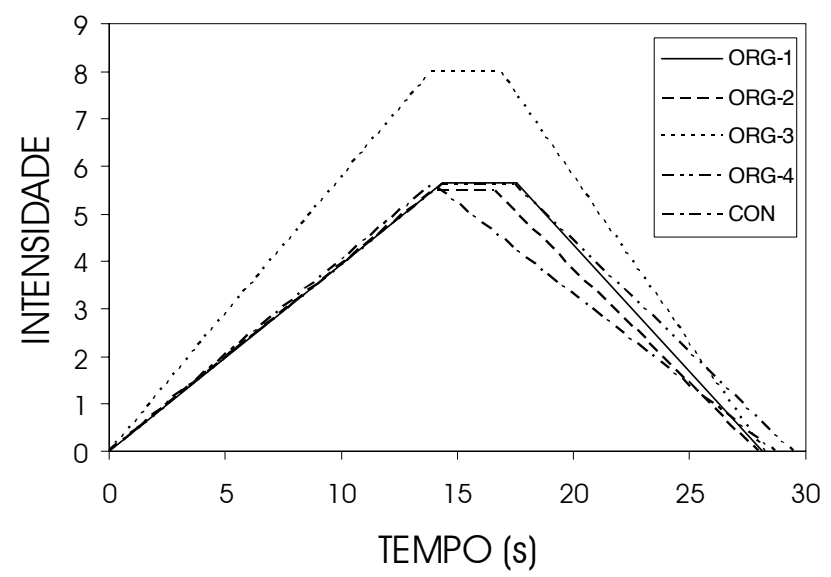

FIGURA 2. Curvas Tempo-Intensidade característica do gosto amargo para as cinco amostras de café
As curvas Tempo-Intensidade característica do gosto amargo para as cinco amostras de café percebidas pelos sete provadores estão apresentadas na Figura 2. A intensidade e a duração da percepção do gosto amargo foi maior para a amostra ORG-3. De acordo com MONTEIRO [10] o tempo de torra influencia na percepção gosto amargo, podendo explicar tal resultado. Por tratar-se de amostras comerciais não houve controle de todas as variáveis e o tempo de torração foi uma delas.

\section{4 - CONCLUSÕES}

A amostra de café orgânico ORG-3 obteve diferença estatística significativa $(\mathrm{p} \leq 0,05)$ em relação as demais amostras de café, em relação aos parâmetros Imax (Intensidade Máxima) e Área (área sob a curva), estando em maior intensidade para o gosto amargo.

As bebidas de café ORG-4 e CON que são da mesma marca não apresentaram diferença significativa $(p \geq 0,05)$ entre si em relação aos seis parâmetros da curva Tempo-Intensidade.

\section{5 - REFERÊNCIAS BIBLIOGRÁFICAS}

[1] BIRCH, G.G.; LATYMER, Z.; HOLLAWAY, M. Intensity/ time relationships in sweetness: Evidence for a queue hypothesis in taste chemoreception. Chemical senses. v. 5. n. 1. p. 63, 1980.

[2] CAIXETA, G.Z.T. Economia Cafeeira: Mercado de Café, Tendências e Perspectivas. Economia Cafeeira Mundial. In: I Encontro sobre produção de café com qualidade, Anais... Viçosa: UFV. 1999. p. 3 -21.

[3] CARDELlO, H.M.A.B.; DAMASIO, M.H. Análise TempoIntensidade. Artigo Técnico: Revisão. Boletim SBCTA. v. 30. n. 2. p. 156-165, 1996.

[4] CARDELLO, H.M.A.B., DA SILVA, M.A.A.P.; DAMASIO, M.H. Análise Tempo-Intensidade dos estímulos doce e amargo de extrato de folha de estévia (Stevia rebaudiana (Bert.) Bertoni) em doçura equivalente a sacarose. Ciênc. Tecnol. Aliment.. v. 19. n. 2. p. 163-169. 1999.

[5] CARDELLO, H.M.A.B.; FARIA, J.B. Análise Tempo-Intensidade de características sensoriais de aguardente de cana durante o envelhecimento em tonel de carvalho (Quercus sp). Boletim SBCTA. v. 33. n. 1. p. 2734, 1999.

[6] CARVAlHO, V.D.; CHAGAS, S.J. de R.; CHALFOUN, S.M. Fatores que afetam a qualidade do café. Informe Agropecuário. v. 18. n. 187. p. 5-20, 1997.

[7] KELLING, S.T., HALPERN, P.B. Taste flashes: reaction times, intensity and quality. Science. v. 219 , p. $412-$ 422, 1983.

[8] LUNDAHL, D.S. Comparing time-intensity to category scales in sensory evaluation. Food Technology, v. 46, n. 11, p. 98-103, 1992.

[9] LEACH, E.J.; NOBLE. A.C. Comparison of bitterness of caffeine and quinine by a time-intensity procedure. Chemical senses. v. 11. p. 339-345, 1986.

[10] MONTEIRO, M.A.M. Caracterização da bebida de café (Coffea arabica L.): Análise Descritiva Quantitativa, Análise Tempo-Intensidade e Testes Afetivos. 2002. 158p. Tese (Doutorado em Ciência e Tecnologia de Alimentos). Universidade Federal de Viçosa (UFV). 
[11] PORTMANN, M.O.; SERGHAT, S.; MATHLOUTHI, M. Study of some factors affecting intensity/time characteristics of sweetness. Food Chemistry. v. 44. p.83-92, 1992.

[12] SAS. SAS Software Version 8. Cary, North Carolina: SAS Institute Inc.. 1999.
[13] THEODORO, V.C.A.; JÚNIOR, M.M.; GUIMARÃES, R.J; CHAGAS, S.J.R. Caracterização da Qualidade de Grãos de Cafés (Coffea arabica L.) Colhidos no Pano e no Chão, Provenientes de Sistemas de Manejo Orgânico, em Conversão e Convencional. Universidade Federal de Lavras. 2002. Disponivel em: <http//www.planetaorganico.com.br/ trabalhos\&pesquisas>. Acessado em: abril de 2002. 